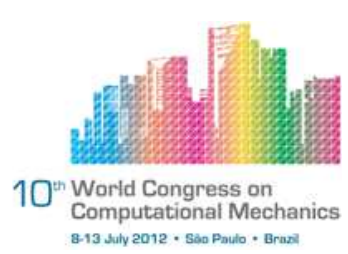

\title{
MULLINS' EFFECT IN SEMICRYSTALLINE POLYMERS: EXPERIMENTS AND MODELINS
}

\author{
A. D. Drozdov ${ }^{1}$, A.-L. Hog Lejre ${ }^{1}$ \\ ${ }^{1}$ Danish Technological Institute (add@teknologisk.dk)
}

\begin{abstract}
Observations are reported on isotactic polypropylene in uniaxial tensile cyclic tests with a mixed program (oscillations between maximum strains $\epsilon_{\max }$ and the zero minimum stress $\sigma_{\min }=0$ when $\epsilon_{\max }$ increase monotonically with number of cycles $n$ ). Experimental data demonstrate fading memory of deformation history: when tensile strain $\epsilon$ exceeds maximum strain at the previous cycle, stress-strain curves become identical for specimens subjected to different loading programs. These observations resemble the Mullins effect: the stress-strain diagram under tension for a specimen subjected to cyclic deformation with maximum strain $\epsilon_{\max }$ rapidly reaches that for a virgin specimen when strain $\epsilon$ exceeds $\epsilon_{\max }$. The difference between our findings and the Mullins phenomenon is that the stress-strain diagrams coincide not only under loading, but under retraction as well, and the coincidence is observed for arbitrary cyclic deformation programs. A constitutive model is derived in cyclic viscoplasticity of semicrystalline polymers with finite strains, and adjustable parameters in the stress-strain relations are found by fitting observations. Ability of the model to predict the mechanical response of polypropylene in multi-cycle tensile tests and to describe the fading memory phenomenon is confirmed by numerical simulation.
\end{abstract}

Keywords: Semicrystalline polymers, Finite viscoplasticity, Cyclic deformation, Mullins effect, Constitutive modeling.

\section{INTRODUCTION}

This paper deals with experimental investigation and constitutive modeling of the mechanical response of isotactic polypropylene in multi-cycle uniaxial tensile tests with a mixed deformation program (oscillations between maximum strains $\epsilon_{\max }$ and the zero minimum stress $\sigma_{\min }=0$ when $\epsilon_{\min }$ increases monotonically with number of cycles $n$ ). Observations reveal the fading memory phenomenon: when strain $\epsilon$ exceeds maximum strain at the previous cycle $\epsilon_{\max }$, the stress-strain diagram becomes independent of deformation history. Unlike the Mullins phenomenon [3] (coincidence of loading paths of stress-strain curves for virgin and pre-loaded specimens at $\epsilon>\epsilon_{\max }$ ), our experimental data demonstrate that unloading paths are identical as well, and the coincidence of 
stress-strain diagrams is not affected by deformation program (stress-controlled, straincontrolled, or mixed), type of loading (tension with a constant strain rate or creep), and conditions of unloading (total retraction to the zero minimum stress or partial retraction to a positive stress).

The experimental data provide a challenge for constitutive modeling: on the one hand, they reveal fading memory of deformation history in cyclic tests with increasing maximum strains, while on the other, they show that under a cycle of loading-retraction down to the zero stress, substantial (comparable with maximum strain under stretching) irreversible strains arise, which means that some memory is preserved in semicrystalline polymers. This implies that conventional stress-strain relations for the description of Mullins' effect $[1,2,4,6-9]$ are inapplicable for the analysis of fading memory, and more sophisticated constitutive equations are required.

The objective of this work is three-fold: (i) to report experimental data on polypropylene in multi-cycle uniaxial tensile tests with mixed deformation program at ambient temperature, (ii) to derive stress-strain relations in cyclic viscoplasticity with finite deformations and to determine adjustable parameters in the constitutive equations by fitting the observations, (iii) to validate ability of the model to describe the fading memory phenomenon and to predict the mechanical behavior of semicrystalline polymers in multi-step cyclic tests by numerical simulation.

A two-step approach is applied for derivation of constitutive equations. At the first step, stress-strain relations are developed for an individual cycle of loadingretraction by means of the Clausius-Duhem inequality. These equations involve several adjustable parameters that are treated as constants along each cycle of oscillations. At the other step, some of these quantities are allowed to change with number of cycles, and phenomenological relations are introduced for their evolution driven by damage accumulation. As a measure of damage, specific plastic work is employed.

The following features of the constitutive model are worth mentioning:

1. Deformation gradient for plastic deformation is split into two components that describe irreversible deformations in the amorphous and crystalline phases.

2. Strain energy density is treated as the sum of mechanical energy stored in the matrix (this energy depends on principal invariants of the Cauchy-Green tensor for elastic deformation) and the energy of interaction between the matrix and crystallites (that depends on principal invariants of the Cauchy-Green tensor for plastic deformation in the matrix).

3. Under multi-cycle tensile deformation with increasing maximum strains $\epsilon_{\max }(n)$, the stress-strain diagram is split into three groups of segments corresponding to (i) stretching (strain $\epsilon$ increases and plastic strain $\epsilon_{\mathrm{p}}$ exceeds maximum plastic strain at the previous cycle $\epsilon_{\mathrm{p} \max }$ ), (ii) unloading (strain decreases), and (iii) reloading ( $\epsilon$ increases, but $\epsilon_{\mathrm{p}}$ remains lower than $\epsilon_{\mathrm{p} \max }$ ).

The exposition is organized as follows. Observations on isotactic polypropylene in multi-step uniaxial tensile cyclic tests at room temperature are reported in Section 


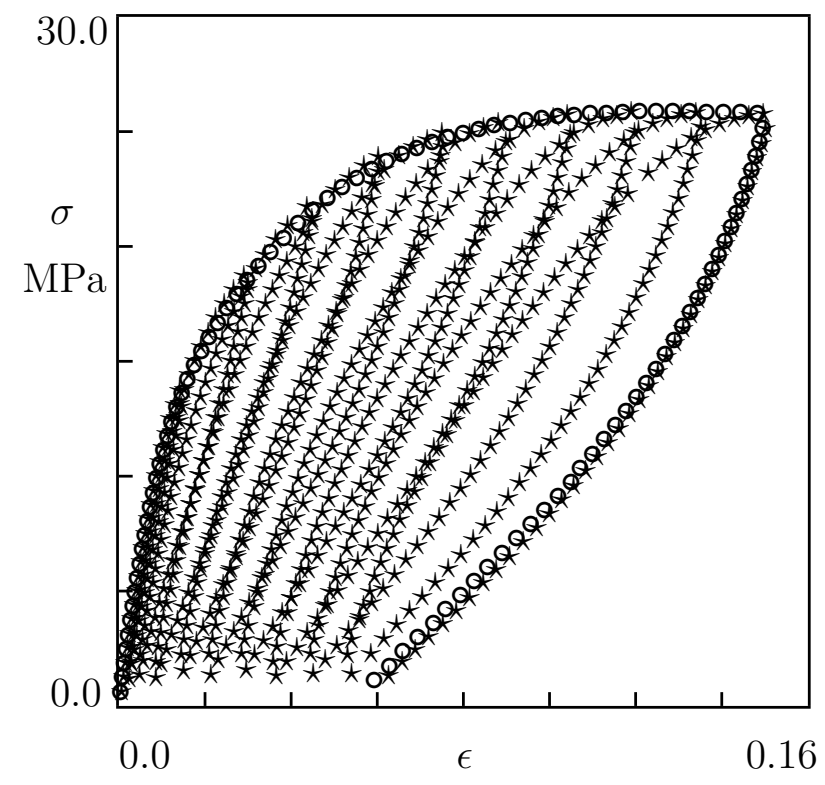

Figure 1: Stress $\sigma$ versus strain $\epsilon$. Symbols: experimental data in cyclic tests (o - T-01; $\star-\mathrm{T}-10)$.

2. Constitutive equations in cyclic viscoplasticity of semicrystalline polymers with finite strains are derived in Section 3. Adjustable parameters in the stress-strain relations are found in Section 4 by fitting the experimental data. Results of numerical simulation are discussed in Section 5. Concluding remarks are formulated in Section 6.

\section{EXPERIMENTAL RESULTS}

Medical grade of isotactic polypropylene Bormed HD $810 \mathrm{MO}$ (density $0.91 \mathrm{~g} / \mathrm{cm}^{3}$, melt flow rate $10 \mathrm{~g} / 10 \mathrm{~min}$ ) was purchased from Borealis AG (Austria). Dumbbell specimens for mechanical tests (ASTM standard D-638) with cross-sectional area $10.1 \mathrm{~mm} \times 4.2$ mm were molded by using injection-molding machine Arburg 320C.

Uniaxial tensile tests were conducted at room temperature by means of universal testing machine Instron-5569 equipped with an electro-mechanical sensor for control of longitudinal strains. Tensile force was measured by $5 \mathrm{kN}$ load cell. The engineering stress $\sigma$ was determined as the ratio of axial force to cross-sectional area of undeformed specimens.

The experimental program involved five series of cyclic tests. Each test was carried out on a new specimen and repeated by twice. Observations revealed good reproducibility of measurements: deviations between stresses measured on different specimens did not exceed $3 \%$. Tests were carried out with cross-head speed $10 \mathrm{~mm} / \mathrm{min}$ that corresponded to strain rate $\dot{\epsilon}=1.7 \cdot 10^{-3} \mathrm{~s}^{-1}$. This strain rate was selected to ensure that maximum strains in each cycle of multi-cycle deformation programs were reproduced with high accuracy (deviations between their real and programmed values did not exceed 0.002).

The first series involved four tests with $N=1,3,5$, and 10 cycles, maximum strains $\epsilon_{\max }(n)=0.15 n / N$, and minimum stress $\sigma_{\min }=1 \mathrm{MPa}$. This stress was chosen 


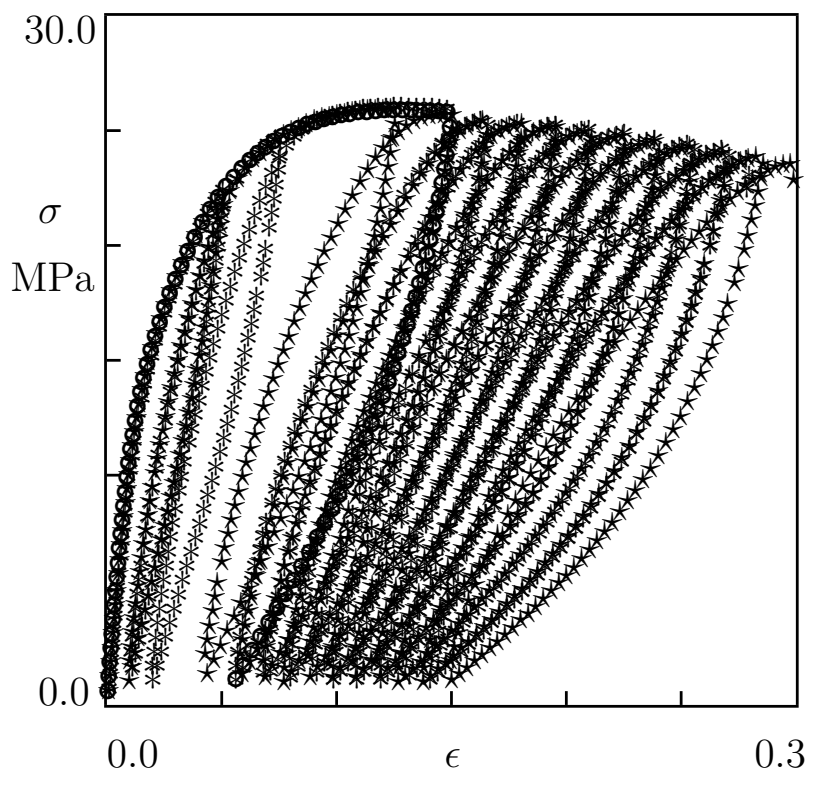

Figure 2: Stress $\sigma$ versus strain $\epsilon$. Symbols: experimental data in cyclic tests (o - T-01; $*-$ TL-075; $\star-$ TL-125).

instead of $\sigma_{\min }=0$ to avoid buckling of specimens. In each test, a specimen was stretched up to the first maximum strain $\epsilon_{\max }(1)$, unloaded down to the minimum stress $\sigma_{\min }$, reloaded up to the second maximum strain $\epsilon_{\max }(2)$, unloaded down to the minimum stress $\sigma_{\min }$, etc. These tests are designated as T-01, T-03, T-05, and T-10, where the last two digits stand for number of cycles.

The second series involved two tests with maximum strains $\epsilon_{\max }(1)=0.05$ and $\epsilon_{\max }(3)=0.15$ (the same as in program T-03), and maximum strains at the second cycle 0.075 and 0.125 . Unlike test T-03, after retraction at the third cycle, cyclic deformations proceeded with increment of maximum strain 0.015 (which means that loadings and unloadings were carried out with maximum strains $\epsilon_{\max }(4)=0.165, \epsilon_{\max }(5)=0.18$, $\epsilon_{\max }(6)=0.195, \epsilon_{\max }(7)=0.21$, etc. up to breakage of specimens which occurred after $N=11$ and 12 cycles, respectively). These tests were designated as TL-075 and TL-125, where the digits denote maximum strains at the second cycle.

Observations in these two series of tests are employed to find material parameters in the stress-strain relations. Other tests were conducted to reveal characteristic features of the fading memory phenomenon.

The third series consisted of a test with $N=3$ cycles, maximum strains $\epsilon_{\max }(n)=$ $0.15 n / N$ and minimum stress $\sigma_{\min }=10 \mathrm{MPa}$. This test was performed to examine the influence of minimum stress on fading of memory and was designated as T-03-10.

The fourth series involved two tests with the following program: stretching up to maximum stress $\sigma_{\max }$, creep flow with this stress for $t_{\mathrm{c}}=5 \mathrm{~min}$, unloading down to minimum stress $\sigma_{\min }=1 \mathrm{MPa}$, reloading up to the same maximum stress $\sigma_{\max }$, creep flow with this stress for $t_{\mathrm{c}}=5 \mathrm{~min}$, unloading down to minimum stress $\sigma_{\min }=1 \mathrm{MPa}$, reloading up to maximum strain $\epsilon_{\max }=0.15$, and retraction down to minimum stress $\sigma_{\min }=1 \mathrm{MPa}$. Cyclic tests interrupted by two intervals of creep were performed with $\sigma_{\max }=15$ and $20 \mathrm{MPa}$. These tests were conducted to evaluate the effect of creep on 


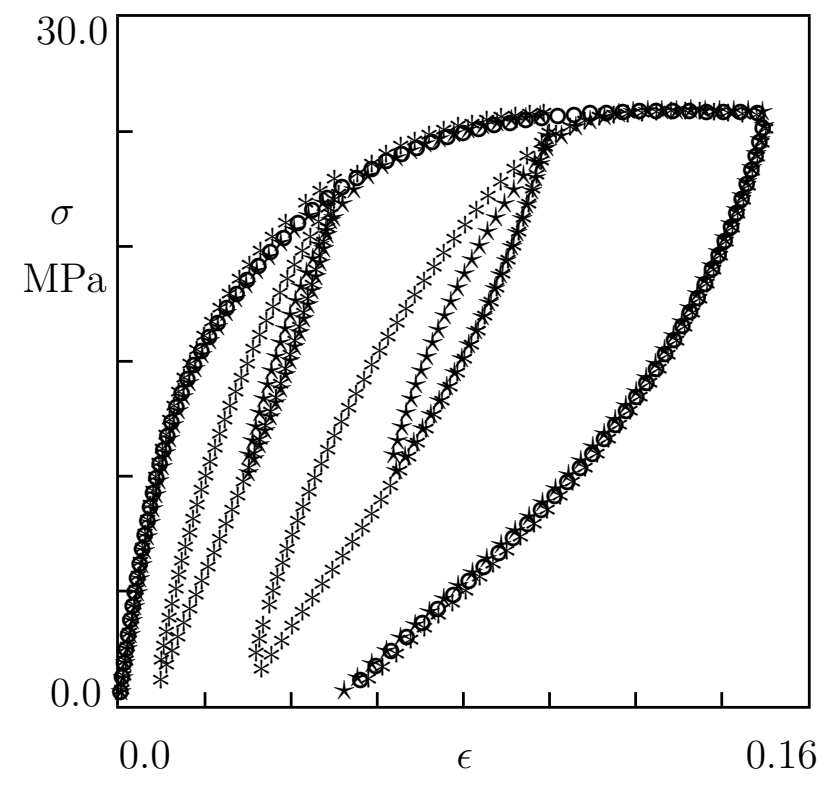

Figure 3: Stress $\sigma$ versus strain $\epsilon$. Symbols: experimental data in cyclic tests (o - T-01; $*-\mathrm{T}-03 ; \star-\mathrm{T}-03-10)$.

the fading memory phenomenon and were designated as TC-15 and TC-20, where the last digits stand for tensile stress at which creep was performed.

The fifth series involved two tests in which specimens were subjected to multicycle deformation with mixed $(N=50$ cycles of oscillations between maximum strain $\epsilon_{\max }=0.05$ and minimum stress $\left.\sigma_{\min }=1 \mathrm{MPa}\right)$ and stress-controlled $(N=100$ cycles of ratcheting between maximum stress $\sigma_{\max }=20 \mathrm{MPa}$ and minimum stress $\sigma_{\min }=1 \mathrm{MPa}$ ) programs. When pre-loading programs were completed, the specimens were immediately stretched up to maximum strain $\epsilon_{\max }=0.15$ and unloaded down to minimum stress $\sigma_{\min }=1 \mathrm{MPa}$. These tests were carried out to examine the influence of cyclic preloading on fading of memory and were designated as TP-050 and TP-100, where the digits denote number of cycles at pre-loading.

The fading memory effect is illustrated in Figures 1-6 where engineering stress $\sigma$ is plotted versus engineering strain $\epsilon$. Figure 1 shows that loading and unloading paths of stress-strain curves coincide along the last cycle of oscillations for a virgin specimen and a sample subjected to 9 cycles of pre-loading with increasing maximum strains. Figure 2 demonstrates that when specimens are subjected to deformation programs that differ along the first $N_{1}=2$ cycles and coincide afterwards, their stress-strain diagrams become identical starting from the $\left(N_{1}+1\right)$ th cycle, and this property is independent of whether cyclic deformation is performed with small or large strains. Figure 3 reveals that coincidence of loading-unloading curves along the last cycle of oscillations occurs independently of whether total (down to $\sigma_{\min }=0$ ) or partial (down to a positive stress $\left.\sigma_{\min }\right)$ retraction was conducted. According to Figure 4, loading and unloading paths of stress-strain curves become identical along the last cycle of oscillations independently of which type of loading (stretching or creep) leads to growth of maximum strains under pre-deformation. Figures 5 and 6 show that cyclic pre-loadings with various programs (mixed and stress-controlled) do not affect coincidence of stress-strain diagrams along 


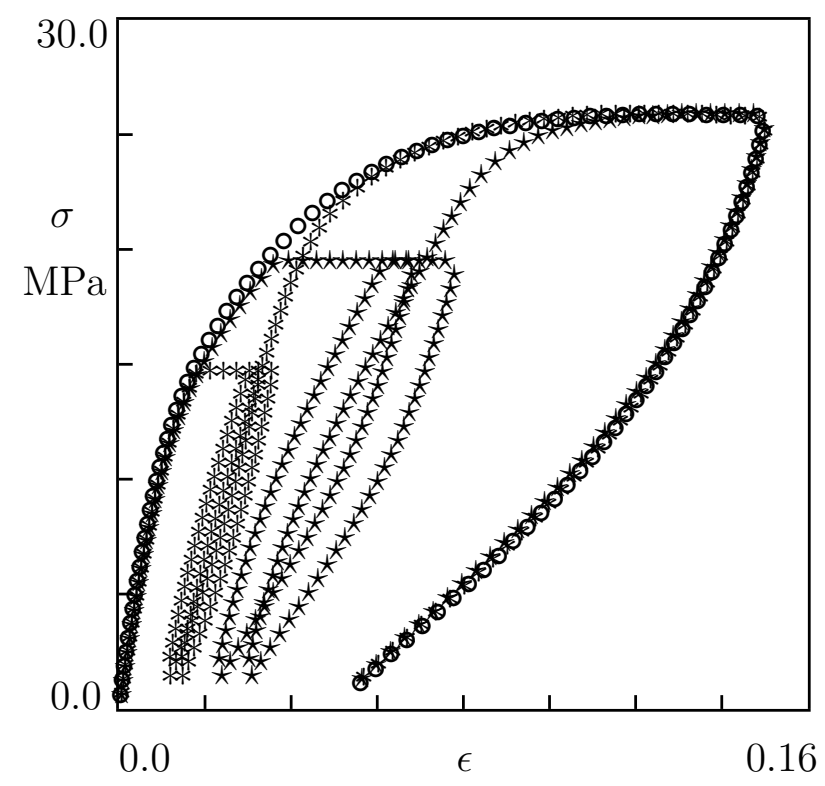

Figure 4: Stress $\sigma$ versus strain $\epsilon$. Symbols: experimental data in cyclic tests (o - T-01; $*-\mathrm{TC}-15 ; \star-\mathrm{TC}-20)$.

the last cycle of deformation.

Experimental stress-strain diagrams in tests T-01, T-03, T-05, T-10, TL-075, and TL-125 are depicted in Figures 7-12. These observations are employed in Section 4 for determination of adjustable parameters in constitutive equations.

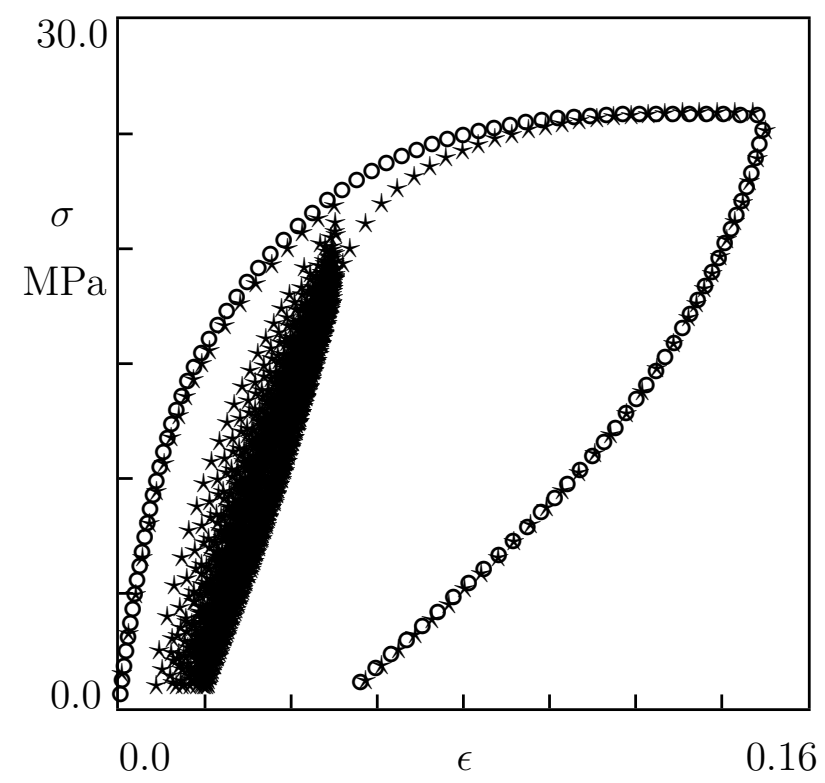

Figure 5: Stress $\sigma$ versus strain $\epsilon$. Symbols: experimental data in cyclic tests (o - T-01; $\star-\mathrm{TP}-050)$. 


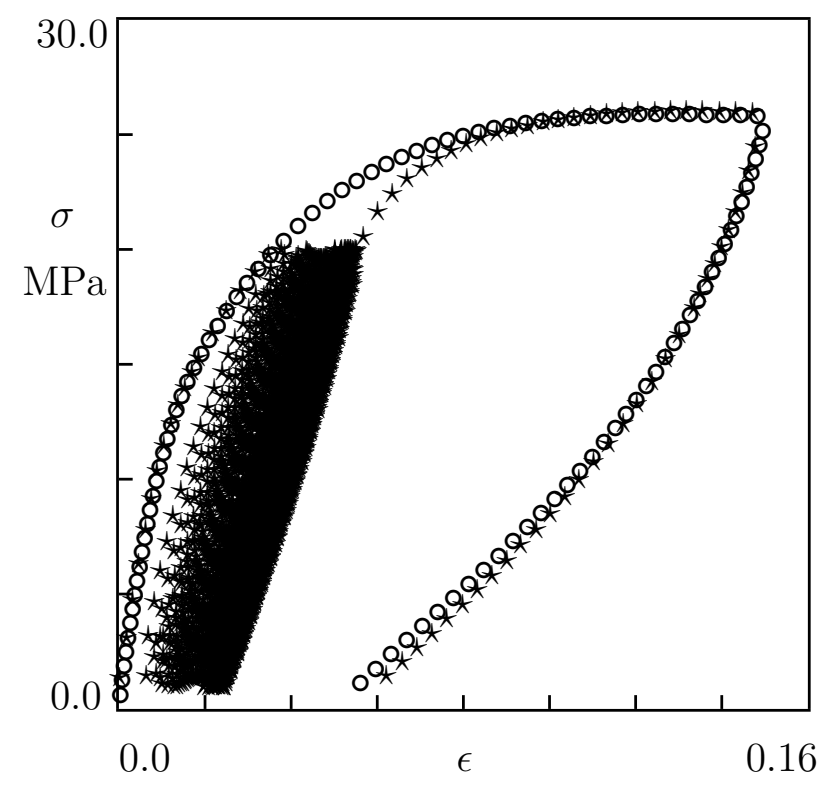

Figure 6: Stress $\sigma$ versus strain $\epsilon$. Symbols: experimental data in cyclic tests (o - T-01; $\star-\mathrm{TP}-100)$.

\section{CONSTITUTIVE MODEL}

Derivation of constitutive equations in cyclic viscoplasticity of semicrystalline polymers is performed within the framework of finite deformations (the linear theory is inapplicable as maximum strains in tests TL-075 and TL-125 reach about 30\%). A two-step approach is applied to develop a constitutive model. At the first step, stress-strain relations are derived for the mechanical response under an arbitrary three-dimensional deformation. At the other step, some parameters in the constitutive equations (that are treated as constants in approximation of a stress-strain diagram for an individual cycle) are allowed to evolve with number of cycles being driven by damage accumulation.

To reduce the number of experimental constants, a semicrystalline polymer is treated as an isotropic, incompressible, viscoplastic medium. With reference to the multiplicative decomposition formula, the deformation gradient for macro-deformation $\mathbf{F}$ is presented in the form

$$
\mathbf{F}=\mathbf{F}_{\mathrm{e}} \cdot \mathbf{F}_{\mathrm{p}}
$$

where $\mathbf{F}_{\mathrm{e}}$ and $\mathbf{F}_{\mathrm{p}}$ are deformation gradients for elastic and plastic deformation, and the dot stands for scalar product. Differentiating Eq. (1) with respect to time $t$ and introducing velocity gradients

$$
\mathbf{L}=\dot{\mathbf{F}} \cdot \mathbf{F}^{-1}, \quad \mathbf{L}_{\mathrm{e}}=\dot{\mathbf{F}}_{\mathrm{e}} \cdot \mathbf{F}_{\mathrm{e}}^{-1}, \quad \mathbf{l}_{\mathrm{p}}=\dot{\mathbf{F}}_{\mathrm{p}} \cdot \mathbf{F}_{\mathrm{p}}^{-1}
$$

we arrive at the formula

$$
\mathbf{L}=\mathbf{L}_{\mathrm{e}}+\mathbf{L}_{\mathrm{p}}, \quad \mathbf{L}_{\mathrm{p}}=\mathbf{F}_{\mathrm{e}} \cdot \mathbf{l}_{\mathrm{p}} \cdot \mathbf{F}_{\mathrm{e}}^{-1}
$$

Tensor $\mathbf{l}_{\mathrm{p}}$ stands for velocity gradient for plastic deformation in the unloaded configuration, and $\mathbf{L}_{\mathrm{p}}$ denotes the same velocity gradient in the actual configuration. Introducing 


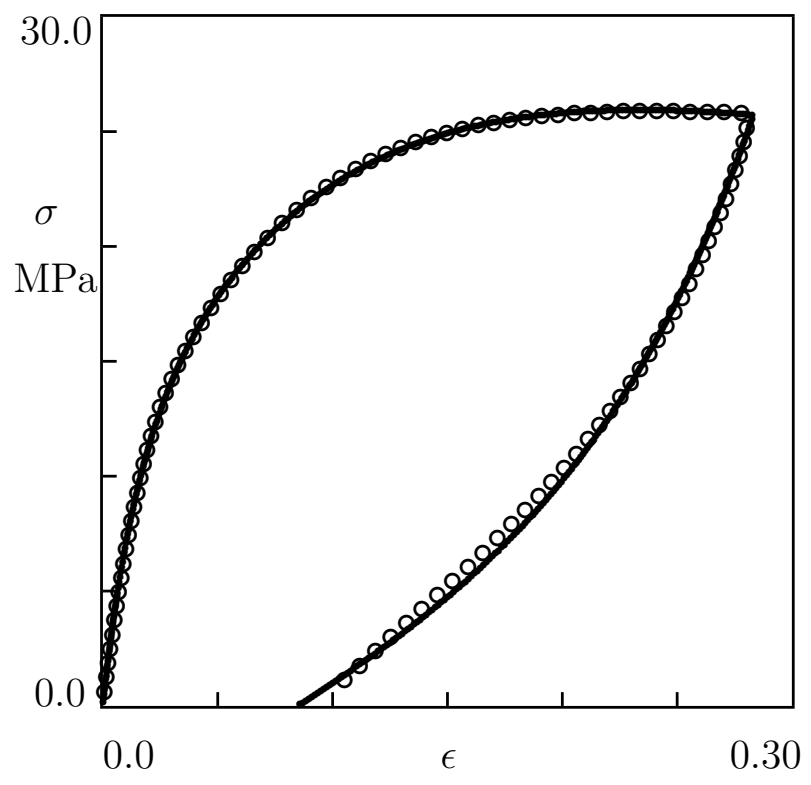

Figure 7: Stress $\sigma$ versus strain $\epsilon$. Circles: experimental data in test T-01. Solid line: results of numerical simulation.

rate-of-strain tensors for macro-deformation $\mathbf{D}$, elastic deformation $\mathbf{D}_{\mathrm{e}}$, and plastic deformation $\mathbf{D}_{\mathrm{p}}$,

$$
\mathbf{D}=\frac{1}{2}\left(\mathbf{L}+\mathbf{L}^{\top}\right), \quad \mathbf{D}_{\mathrm{e}}=\frac{1}{2}\left(\mathbf{L}_{\mathrm{e}}+\mathbf{L}_{\mathrm{e}}^{\top}\right), \quad \mathbf{D}_{\mathrm{p}}=\frac{1}{2}\left(\mathbf{L}_{\mathrm{p}}+\mathbf{L}_{\mathrm{p}}^{\top}\right),
$$

where $T$ stands for transpose, we find from Eq. (3) that

$$
\mathrm{D}=\mathrm{D}_{\mathrm{e}}+\mathrm{D}_{\mathrm{p}}
$$

where

$$
\mathbf{D}_{\mathrm{p}}=\frac{1}{2}\left(\mathbf{F}_{\mathrm{e}} \cdot \mathbf{l}_{\mathrm{p}} \cdot \mathbf{F}_{\mathrm{e}}^{-1}+\mathbf{F}_{\mathrm{e}}^{-\top} \cdot \mathbf{l}_{\mathrm{p}}^{\top} \cdot \mathbf{F}_{\mathrm{e}}^{\top}\right)
$$

To account for inhomogeneity of a semicrystalline polymer (where spherulites are randomly distributed in an amorphous matrix), the deformation gradient for plastic deformation $\mathbf{F}_{\mathrm{p}}$ should be split into two components, $\mathbf{F}_{\mathrm{pa}}$ and $\mathbf{F}_{\mathrm{pc}}$, that characterize plastic flow in the amorphous and crystalline phases, respectively. When one of these phases governs plastic deformation in the other, this can be easily done by setting $\mathbf{F}_{\mathrm{p}}=\mathbf{F}_{\mathrm{pa}} \cdot \mathbf{F}_{\mathrm{pc}}$ or $\mathbf{F}_{\mathrm{p}}=\mathbf{F}_{\mathrm{pc}} \cdot \mathbf{F}_{\mathrm{pa}}$ following Eq. (1). As no experimental data are available for semicrystalline polymers confirming subordination of plastic deformation in one phase with respect to that in the other, plastic flows in these phases are treated as independent. To express $\mathbf{F}_{\mathrm{p}}$ in terms of $\mathbf{F}_{\mathrm{pa}}$ and $\mathbf{F}_{\mathrm{pc}}$, it is postulated that the velocity gradient $\mathbf{l}_{\mathrm{p}}$ equals the sum of velocity gradients for corresponding plastic deformations

$$
\mathrm{l}_{\mathrm{p}}=\mathrm{l}_{\mathrm{pa}}+\mathrm{l}_{\mathrm{pc}}
$$

Under the conventional hypothesis that the plastic spins vanish,

$$
\mathrm{l}_{\mathrm{pa}}=\mathrm{l}_{\mathrm{pa}}^{\top}=\mathrm{d}_{\mathrm{pa}}, \quad \mathrm{l}_{\mathrm{pc}}=\mathrm{l}_{\mathrm{pc}}^{\top}=\mathrm{d}_{\mathrm{pc}},
$$




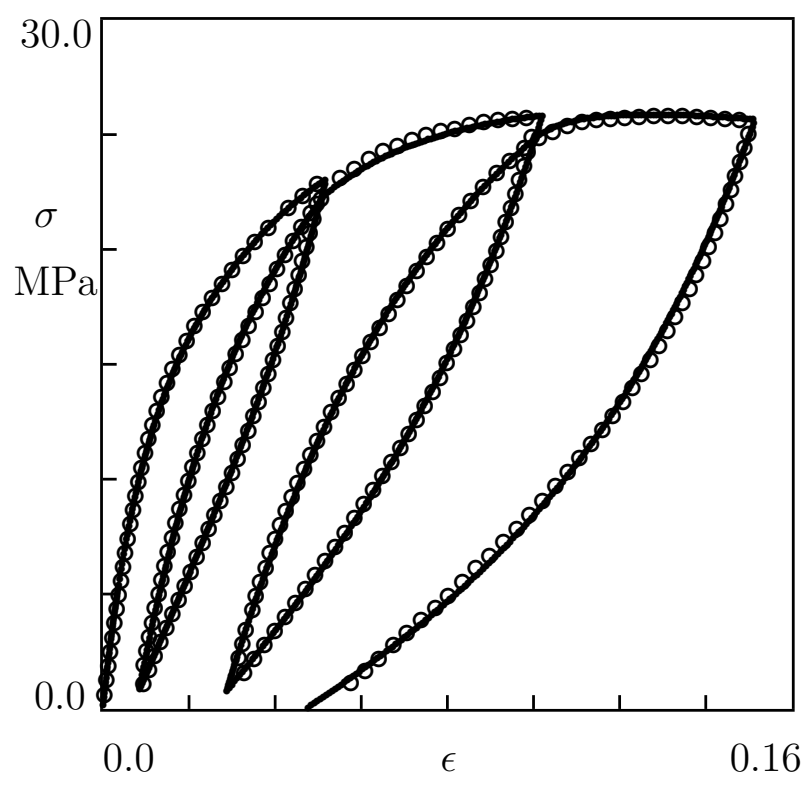

Figure 8: Stress $\sigma$ versus strain $\epsilon$. Circles: experimental data in test T-03. Solid line: results of numerical simulation.

we find from Eq. (7) that

$$
\mathrm{l}_{\mathrm{p}}=\mathrm{l}_{\mathrm{p}}^{\top}=\mathrm{d}_{\mathrm{p}} .
$$

Combination of Eqs. (6)-(9) implies that

$$
\mathbf{D}_{\mathrm{p}}=\mathbf{D}_{\mathrm{pa}}+\mathbf{D}_{\mathrm{pc}}
$$

where

$$
2 \mathbf{D}_{\mathrm{pa}}=\mathbf{F}_{\mathrm{e}} \cdot \mathbf{d}_{\mathrm{pa}} \cdot \mathbf{F}_{\mathrm{e}}^{-1}+\mathbf{F}_{\mathrm{e}}^{-\top} \cdot \mathbf{d}_{\mathrm{pa}} \cdot \mathbf{F}_{\mathrm{e}}^{\top}, \quad 2 \mathbf{D}_{\mathrm{pc}}=\mathbf{F}_{\mathrm{e}} \cdot \mathbf{d}_{\mathrm{pc}} \cdot \mathbf{F}_{\mathrm{e}}^{-1}+\mathbf{F}_{\mathrm{e}}^{-\top} \cdot \mathbf{d}_{\mathrm{pc}} \cdot \mathbf{F}_{\mathrm{e}}^{\top} \cdot
$$

Introducing the Cauchy-Green tensors for elastic deformation

$$
\mathbf{B}_{\mathrm{e}}=\mathbf{F}_{\mathrm{e}} \cdot \mathbf{F}_{\mathrm{e}}^{\top}, \quad \mathbf{C}_{\mathrm{e}}=\mathbf{F}_{\mathrm{e}}^{\top} \cdot \mathbf{F}_{\mathrm{e}}
$$

we find from Eq. (11) that

$$
\mathbf{C}_{\mathrm{e}} \cdot \mathbf{d}_{\mathrm{pa}}+\mathbf{d}_{\mathrm{pa}} \cdot \mathbf{C}_{\mathrm{e}}=2 \mathbf{F}_{\mathrm{e}}^{\top} \cdot \mathbf{D}_{\mathrm{pa}} \cdot \mathbf{F}_{\mathrm{e}}, \quad \mathbf{C}_{\mathrm{e}} \cdot \mathbf{d}_{\mathrm{pc}}+\mathbf{d}_{\mathrm{pc}} \cdot \mathbf{C}_{\mathrm{e}}=2 \mathbf{F}_{\mathrm{e}}^{\top} \cdot \mathbf{D}_{\mathrm{pc}} \cdot \mathbf{F}_{\mathrm{e}}
$$

Given a velocity gradient $\mathbf{l}_{\mathrm{pa}}$, the corresponding deformation gradient $\mathbf{F}_{\mathrm{pa}}$ is defined as a solution of the differential equation

$$
\dot{\mathbf{F}}_{\mathrm{pa}}=\mathbf{l}_{\mathrm{pa}} \cdot \mathbf{F}_{\mathrm{pa}}
$$

with the initial condition $\mathbf{F}_{\mathrm{pa}}(0)=\mathbf{I}$, where $\mathbf{I}$ stands for the unit tensor. Setting

$$
\mathbf{B}_{\mathrm{pa}}=\mathbf{F}_{\mathrm{pa}} \cdot \mathbf{F}_{\mathrm{pa}}^{\top}
$$

and differentiating Eqs. (12) and (15) with respect to time, we find from Eqs. (2), (8), (14) that

$$
\dot{\mathbf{B}}_{\mathrm{e}}=\mathbf{L}_{\mathrm{e}} \cdot \mathbf{B}_{\mathrm{e}}+\mathbf{B}_{\mathrm{e}} \cdot \mathbf{L}_{\mathrm{e}}^{\top}, \quad \dot{\mathbf{B}}_{\mathrm{pa}}=\mathbf{d}_{\mathrm{pa}} \cdot \mathbf{B}_{\mathrm{pa}}+\mathbf{B}_{\mathrm{pa}} \cdot \mathbf{d}_{\mathrm{pa}} \cdot
$$




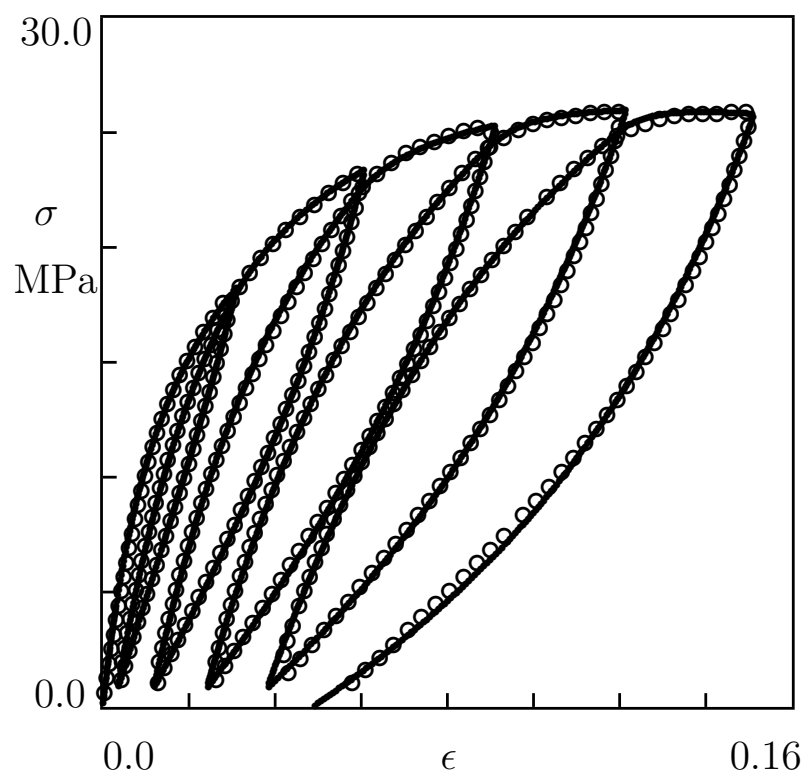

Figure 9: Stress $\sigma$ versus strain $\epsilon$. Circles: experimental data in test T-05. Solid line: results of numerical simulation.

The first principal invariants of the Cauchy-Green tensors $\mathbf{B}_{\mathrm{e}}$ and $\mathbf{B}_{\mathrm{pa}}$ read

$$
J_{1 \mathrm{e}}=\mathbf{B}_{\mathrm{e}}: \mathbf{I}, \quad J_{1 \mathrm{pa}}=\mathbf{B}_{\mathrm{pa}}: \mathbf{I}
$$

where the colon denotes convolution. Differentiating Eq. (17) with respect to time and using Eqs. (5), (10), (13), (16), we arrive at the formulas

$$
\dot{J}_{1 \mathrm{e}}=2 \mathbf{B}_{\mathrm{e}}:\left(\mathbf{D}-\mathbf{D}_{\mathrm{pc}}\right)-2 \mathbf{C}_{\mathrm{e}}: \mathbf{d}_{\mathrm{pa}}, \quad \dot{J}_{1 \mathrm{pa}}=2 \mathbf{B}_{\mathrm{pa}}: \mathbf{d}_{\mathrm{pa}} .
$$

The following hypotheses are introduced:

1. Strain energy density $W$ equals the sum of strain energy density of the amorphous matrix (that depends on the first principal invariant $J_{1 \mathrm{e}}$ of the Cauchy-Green tensor for elastic deformation) and the energy of interaction between polymer chains and crystallites (that depends on the first principal invariant $J_{1 \mathrm{pa}}$ of the CauchyGreen tensor for plastic deformation in the matrix). Adopting neo-Hookean expressions for both functions, we write

$$
W=\frac{1}{2} \mu\left(J_{1 \mathrm{e}}-3\right)+\frac{1}{2} \tilde{\mu}\left(J_{1 \mathrm{pa}}-3\right),
$$

where $\mu$ and $\tilde{\mu}$ stand for elastic moduli.

2. The rate-of-strain tensor for plastic deformation in crystallites $\mathbf{D}_{\mathrm{pc}}$ is proportional to that for macro-deformation $\mathbf{D}$

$$
\mathbf{D}_{\mathrm{pc}}=\phi \mathbf{D}
$$

where $\phi$ is a non-negative scalar function. 


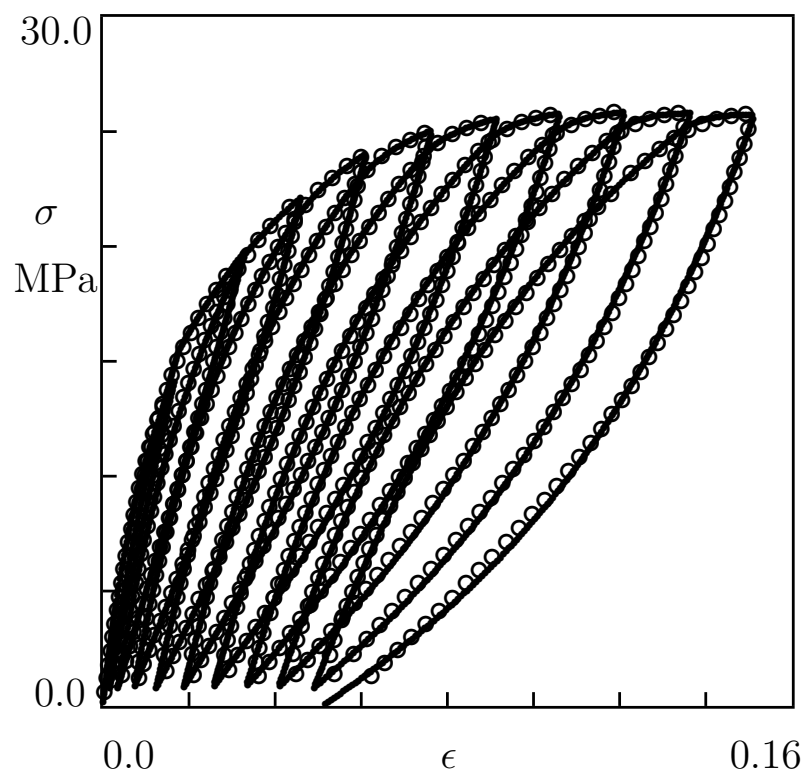

Figure 10: Stress $\sigma$ versus strain $\epsilon$. Circles: experimental data in test T-10. Solid line: results of numerical simulation.

Differentiating Eq. (19) with respect to time and using Eqs. (18), (20), we find that

$$
\dot{W}=\mu\left[(1-\phi) \mathbf{B}_{\mathrm{e}}: \mathbf{D}-\left(\mathbf{C}_{\mathrm{e}}-R \mathbf{B}_{\mathrm{pa}}\right): \mathbf{d}_{\mathrm{pa}}\right]
$$

with $R=\tilde{\mu} / \mu$.

The Clausius-Duhem inequality for isothermal deformation of an incompressible medium reads

$$
Q=-\dot{W}+\Sigma^{\prime}: \mathbf{D} \geq 0,
$$

where $Q$ stands for internal dissipation per unit volume and unit time, $\boldsymbol{\Sigma}$ is the Cauchy stress tensor, and the prime stands for the deviatoric component of a tensor. Inserting Eq. (21) into Eq. (22) and bearing in mind that $\mathbf{D}$ and $\mathbf{d}_{\mathrm{pa}}$ are traceless tensors, we find that Eq. (22) is satisfied for an arbitrary deformation program, provided that (i) the Cauchy stress tensor reads

$$
\Sigma=-p \mathbf{I}+\mu(1-\phi) \mathbf{B}_{\mathrm{e}}
$$

where $p$ denotes an unknown pressure, and (ii) the rate of strain tensor in the matrix $\mathbf{d}_{\mathrm{pa}}$ obeys the equation

$$
\mathbf{d}_{\mathrm{pa}}=\frac{1}{2} P\left[\mathbf{C}_{\mathrm{e}}-R \mathbf{B}_{\mathrm{pa}}-\frac{1}{3}\left(J_{1 \mathrm{e}}-R J_{1 \mathrm{pa}}\right) \mathbf{I}\right],
$$

where $P$ is an arbitrary non-negative function.

Insertion of Eq. (24) into Eq. (16) yields

$$
\dot{\mathbf{B}}_{\mathrm{pa}}=P\left[\frac{1}{2}\left(\mathbf{C}_{\mathrm{e}} \cdot \mathbf{B}_{\mathrm{pa}}+\mathbf{B}_{\mathrm{pa}} \cdot \mathbf{C}_{\mathrm{e}}\right)-\frac{1}{3}\left(J_{1 \mathrm{e}}-R J_{2 \mathrm{pa}}\right) \mathbf{B}_{\mathrm{pa}}-R \mathbf{B}_{\mathrm{pa}}^{2}\right] .
$$

Differentiating Eq. (12) with respect to time and using Eqs. (2), (4), (5), (10), (13), (20), (24), we find that

$$
\dot{\mathbf{C}}_{\mathrm{e}}=2(1-\phi) \mathbf{F}_{\mathrm{e}}^{\top} \cdot \mathbf{D} \cdot \mathbf{F}_{\mathrm{e}}+P\left[\frac{1}{2} R\left(\mathbf{B}_{\mathrm{pa}} \cdot \mathbf{C}_{\mathrm{e}}+\mathbf{C}_{\mathrm{e}} \cdot \mathbf{B}_{\mathrm{pa}}\right)+\frac{1}{3}\left(J_{1 \mathrm{e}}-R J_{1 \mathrm{pa}}\right) \mathbf{C}_{\mathrm{e}}-\mathbf{C}_{\mathrm{e}}^{2}\right] .
$$




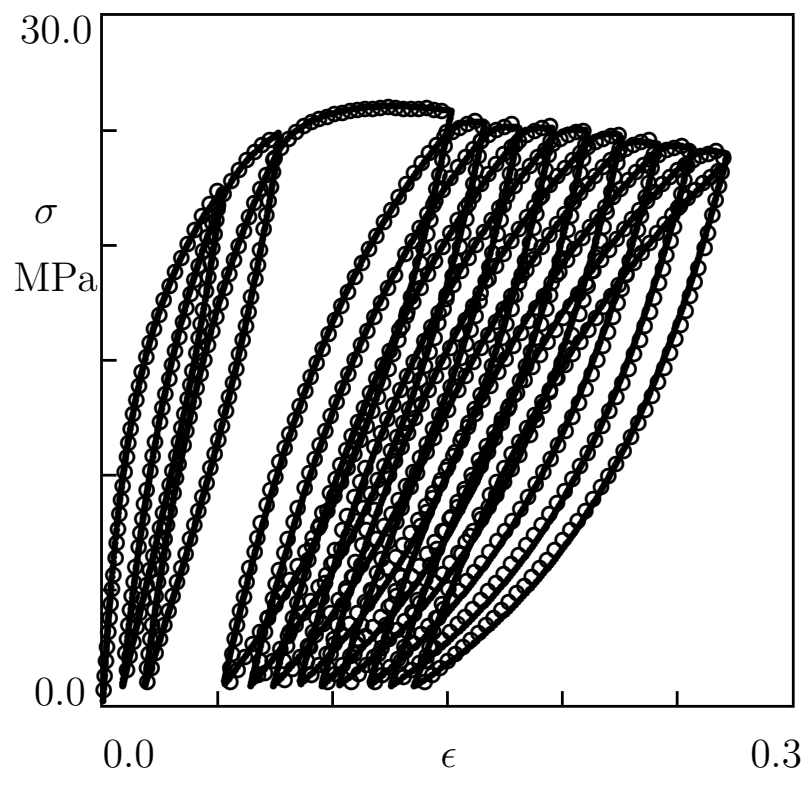

Figure 11: Stress $\sigma$ versus strain $\epsilon$. Circles: experimental data in test TL-075. Solid line: results of numerical simulation.

For an arbitrary three-dimensional deformation, Eqs. (23), (25), and (26) should be accompanied by kinematic equations for tensors $\mathbf{F}_{\mathrm{e}}$ and $\mathbf{B}_{\mathrm{e}}$. For deformations without rotation (in particular, for uniaxial tension, which is the subject of experimental investigation), these equations form a closed set of stress-strain relations.

Uniaxial tension of an incompressible medium is described by the formulas

$$
x_{1}=k X_{1}, \quad x_{2}=k^{-\frac{1}{2}} X_{2}, \quad x_{3}=k^{-\frac{1}{2}} X_{3},
$$

where $\left\{X_{k}\right\}$ and $\left\{x_{k}\right\}$ are Cartesian coordinates in the reference and actual states, and $k=k(t)$ stands for elongation ratio. It follows from Eq. (27) that

$$
\begin{aligned}
\mathbf{F} & =k \mathbf{e}_{1} \mathbf{e}_{1}-k^{-\frac{1}{2}}\left(\mathbf{e}_{2} \mathbf{e}_{2}+\mathbf{e}_{3} \mathbf{e}_{3}\right), \\
\mathbf{B} & =\mathbf{C}=k^{2} \mathbf{e}_{1} \mathbf{e}_{1}-k^{-1}\left(\mathbf{e}_{2} \mathbf{e}_{2}+\mathbf{e}_{3} \mathbf{e}_{3}\right), \\
\mathbf{D} & =\frac{\dot{k}}{k}\left[\mathbf{e}_{1} \mathbf{e}_{1}-\frac{1}{2}\left(\mathbf{e}_{2} \mathbf{e}_{2}+\mathbf{e}_{3} \mathbf{e}_{3}\right)\right],
\end{aligned}
$$

where $\mathbf{e}_{m}(m=1,2,3)$ are base vectors of the Cartesian frame in the reference state. Assuming tensors $\mathbf{F}_{\mathrm{e}}$ and $\mathbf{F}_{\mathrm{pa}}$ to be determined by Eq. (28) with coefficients $k_{\mathrm{e}}$ and $k_{\text {pa }}$, respectively, and inserting these relations into Eqs. (25) and (26), we arrive at the equations

$$
\begin{aligned}
\dot{k}_{\mathrm{pa}} & =\frac{P}{3}\left[\frac{k_{\mathrm{pa}}}{k_{\mathrm{e}}}\left(k_{\mathrm{e}}^{3}-1\right)-R\left(k_{\mathrm{pa}}^{3}-1\right)\right], \\
\dot{k}_{\mathrm{e}} & =(1-\phi) \dot{k} \frac{k_{\mathrm{e}}}{k}-\frac{P}{3} \frac{k_{\mathrm{e}}}{k_{\mathrm{pa}}}\left[\frac{k_{\mathrm{pa}}}{k_{\mathrm{e}}}\left(k_{\mathrm{e}}^{3}-1\right)-R\left(k_{\mathrm{pa}}^{3}-1\right)\right] .
\end{aligned}
$$

Substituting Eq. (28) for $\mathbf{B}_{\mathrm{e}}$ into Eq. (23) and taking into account that under uniaxial tension, the Cauchy stress tensor reads $\Sigma=\Sigma \mathbf{e}_{1} \mathbf{e}_{1}$, we find that the engineering stress $\sigma=\Sigma / k$ is given by

$$
\sigma=\mu(1-\phi) \frac{k_{\mathrm{e}}^{3}-1}{k k_{\mathrm{e}}}
$$




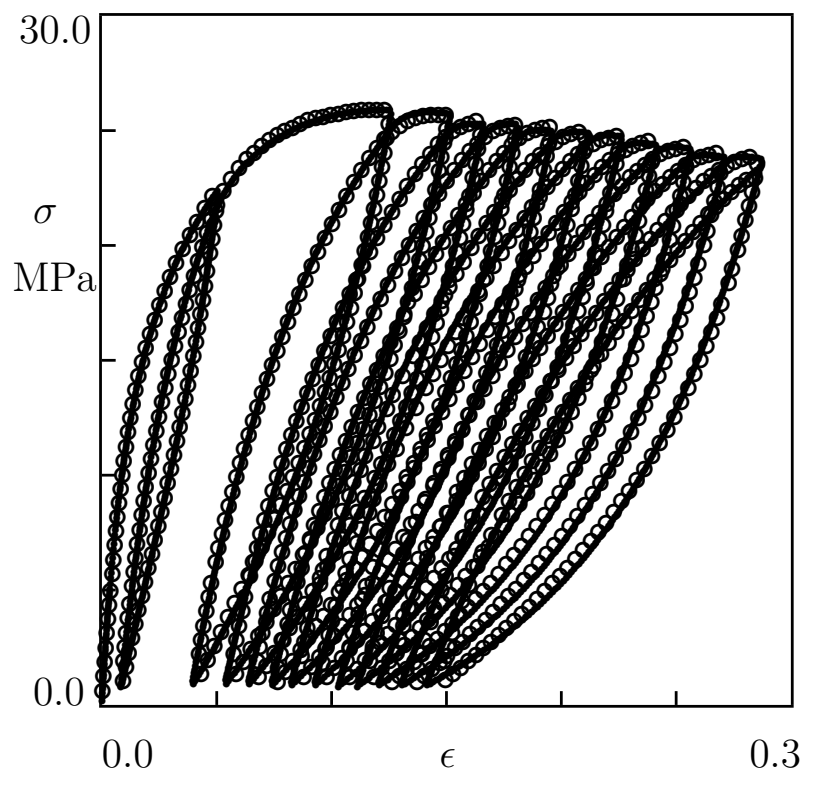

Figure 12: Stress $\sigma$ versus strain $\epsilon$. Circles: experimental data in test TL-125. Solid line: results of numerical simulation.

Equations (29) and (30) provide stress-strain relations for uniaxial tension of semicrystalline polymers with an arbitrary program $k(t)$. These equations involve one material parameter $\mu$ and three adjustable functions, $\phi, P$, and $R$. The following difference between these function is to be mentioned: (i) $P$ is included into kinematic equations (29) only, which means that it can accept arbitrary values when $\dot{k}$ is positive or negative, (ii) $\phi$ is also included into Eq. (30), which implies that it ought to be a continuous function of elongation ratio $k$, (iii) in the derivation of the constitutive equations, coefficient $R$ was treated as a constant, which means that it can adopt different, but constant values along tension and retraction paths.

To introduce kinetic equations for the material functions, the entire stress-strain diagram for cyclic deformation is split into three groups of intervals corresponding to (i) stretching ( $k$ increases and elongation ratio for plastic deformation $k_{\mathrm{p}}=k / k_{\mathrm{e}}$ exceeds its maximum value $k_{\mathrm{p} \max }(n-1)$ at the previous cycle of oscillations), (ii) unloading ( $k$ decreases), and (iii) reloading ( $k$ increases, but $k_{\mathrm{p}}$ remains lower than $\left.k_{\mathrm{p} \max }(n-1)\right)$. According to this approach, the first cycle of deformation involves only stretching up to the first maximum elongation ratio $k_{\max }(1)$. The other cycle consists of unloading down to $\sigma_{\min }$, reloading from $\sigma_{\min }$ up to the point where $k_{\mathrm{p}}=k_{\mathrm{p} \max }(1)$, and stretching up to $k_{\max }(2)$. Unloading, reloading, and stretching for subsequent cycles of deformation are defined in a similar manner.

Confining ourselves to the analysis of cyclic tests with a constant cross-head speed $(|\dot{k}|$ remains constant) and introducing the notation $S=P /|\dot{k}|$, we present Eqs. (29) in the form

$$
\begin{aligned}
\frac{\mathrm{d} k_{\mathrm{pa}}}{\mathrm{d} k} & = \pm \frac{S}{3}\left[\frac{k_{\mathrm{pa}}}{k_{\mathrm{e}}}\left(k_{\mathrm{e}}^{3}-1\right)-R\left(k_{\mathrm{pa}}^{3}-1\right)\right], \\
\frac{\mathrm{d} k_{\mathrm{e}}}{\mathrm{d} k} & =(1-\phi) \frac{k_{\mathrm{e}}}{k} \mp \frac{S}{3} \frac{k_{\mathrm{e}}}{k_{\mathrm{pa}}}\left[\frac{k_{\mathrm{pa}}}{k_{\mathrm{e}}}\left(k_{\mathrm{e}}^{3}-1\right)-R\left(k_{\mathrm{pa}}^{3}-1\right)\right],
\end{aligned}
$$


where the sign "+" corresponds to intervals with $\dot{k}>0$, the sign "_" corresponds to intervals with $\dot{k}<0$, and the coefficients $R$ and $S$ adopt non-negative values $R_{1}, R_{2}, R_{3}$ and $S_{1}, S_{2}, S_{3}$ under stretching, unloading, and reloading, respectively.

Evolution of the function $\phi$ is governed by the differential equation

$$
\dot{\phi}=A(1-\phi)^{2}
$$

with the initial condition $\phi(0)=0$. The coefficient $A$ in Eq. (32) adopts non-negative values $A_{1}, A_{2}, A_{3}$ along intervals of stretching, unloading, and reloading. To reduce the number of material constants, we suppose that changes in $\phi$ occur under stretching only $\left(A_{2}=A_{3}=0\right)$, which implies that Eq. (32) is presented in the form

$$
\frac{\mathrm{d} \phi}{\mathrm{d} k}=a_{1}(1-\phi)^{2} \quad \text { (stretching) }, \quad \frac{\mathrm{d} \phi}{\mathrm{d} k}=0 \quad \text { (unloading and reloading) }
$$

with $a_{1}=A_{1} /|\dot{k}|$.

Stress-strain relations for cyclic tensile deformation with finite strains (30), (31), (33) involve eight parameters: $\mu, a_{1}, R_{1}, S_{1}$ (stretching), $R_{2}, S_{2}$ (unloading), and $R_{3}$, $S_{3}$ (reloading). To minimize the number of experimental constants, we presume that

1. Coefficients $\mu, a_{1}, S_{1}$ are material constants (these quantities accept the same values for each cycle of oscillations).

2. Coefficients $R_{1}, R_{2}, R_{3}$ and $S_{2}, S_{3}$ are uniquely determined by specific plastic work $W_{\mathrm{p}}$ at the instants when corresponding intervals of stretching, unloading and reloading start.

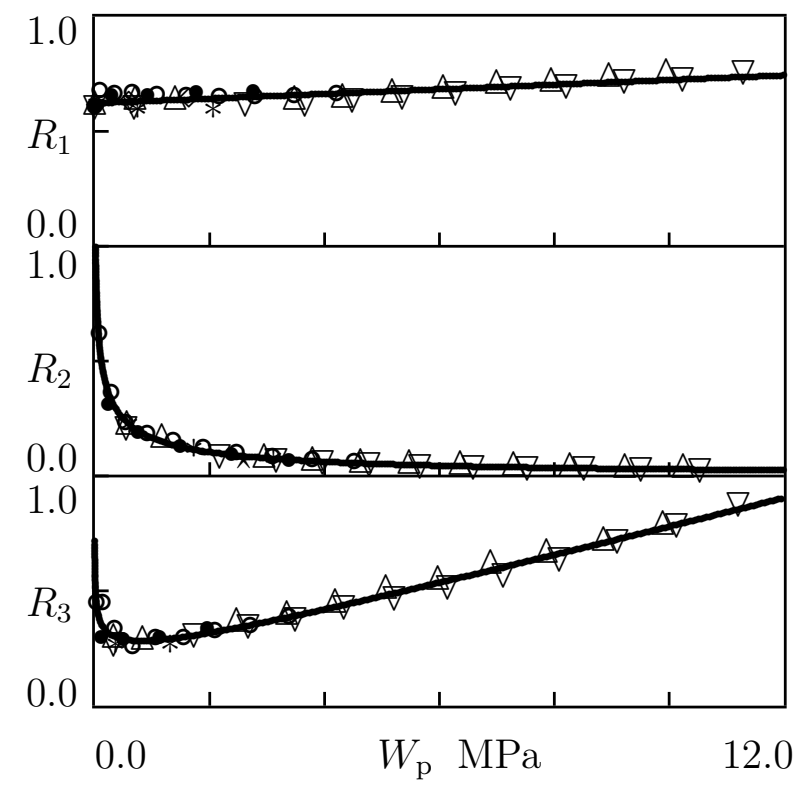

Figure 13: Parameters $R_{1}, R_{2}, R_{3}$ versus plastic work $W_{\mathrm{p}}$. Symbols: treatment of observations in cyclic tests $(\circ-\mathrm{T}-10 ; \bullet-\mathrm{T}-05 ; *-\mathrm{T}-03 ; \star-\mathrm{T}-01 ; \Delta-\mathrm{TL}-075 ; \nabla-$ TL-125). Solid lines: approximation of the data by Eq. (35). 


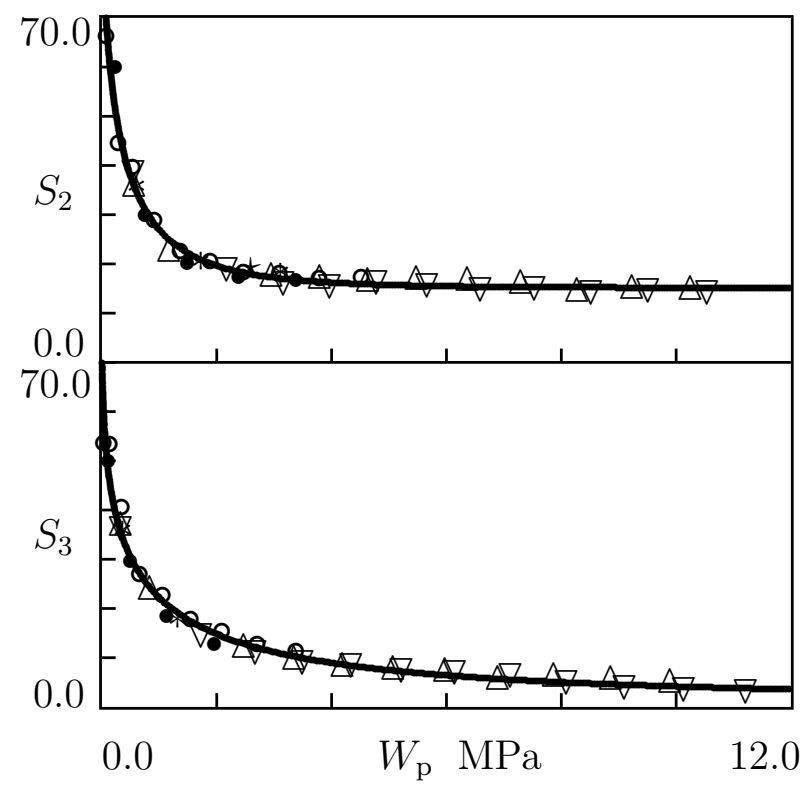

Figure 14: Parameters $S_{2}, S_{3}$ versus plastic work $W_{\mathrm{p}}$. Symbols: treatment of observations in cyclic tests $(\circ-\mathrm{T}-10 ; \bullet-\mathrm{T}-05 ; *-\mathrm{T}-03 ; \star-\mathrm{T}-01 ; \Delta-\mathrm{TL}-075 ; \nabla-\mathrm{TL}-125)$. Solid lines: approximation of the data by Eq. (36).

For an arbitrary three-dimensional deformation, the specific plastic work $W_{\mathrm{p}}$ is defined as a solution of the differential equation

$$
\dot{W}_{\mathrm{p}}=\boldsymbol{\Sigma}^{\prime}: \mathbf{D}_{\mathrm{p}}
$$

with the initial condition $W_{\mathrm{p}}(0)=0$. Under uniaxial tension, this equation is simplified

$$
\dot{W}_{\mathrm{p}}=\sigma\left(\phi \dot{k}+\frac{k}{k_{\mathrm{pa}}} \dot{k}_{\mathrm{pa}}\right) .
$$

The effect of $W_{\mathrm{p}}$ on parameters $R_{i}$ and $S_{i}$ is described by the phenomenological relations

$$
\begin{aligned}
& R_{1}=R_{1}^{0}+R_{1}^{2} W_{\mathrm{p}}, \\
& R_{2}=R_{2}^{0}+R_{2}^{1} \exp \left(-\alpha_{R 2} W_{\mathrm{p}}^{\beta_{R 2}}\right), \\
& R_{3}=R_{3}^{0}+R_{3}^{1} \exp \left(-\alpha_{R 3} W_{\mathrm{p}}^{\beta_{R 3}}\right)+R_{3}^{2} W_{\mathrm{p}}
\end{aligned}
$$

and

$$
S_{2}=S_{2}^{0}+S_{2}^{1} \exp \left(-\alpha_{S 2} W_{\mathrm{p}}^{\beta_{S 2}}\right), \quad S_{3}=S_{3}^{0}+S_{3}^{1} \exp \left(-\alpha_{S 3} W_{\mathrm{p}}^{\beta_{S 3}}\right),
$$

Equations (35) and (36) imply that $R_{2}, S_{2}$, and $S_{3}$ decrease with number of cycles. This decay is attributed to damage accumulation in the amorphous phase (nucleation and growth of micro-voids and micro-cracks). On the contrary, parameters $R_{1}$ and $R_{3}$ increase with number of cycles (at least, after an initial period of decay). This increase is associated with damage growth in the crystalline phase (fragmentation of weak lamellae and alignment of broken lamellar pieces along the loading direction [5]).

\section{FITTING OF OBSERVATIONS}

To find adjustable parameters in the constitutive equations, we approximate the experimental data depicted in Figures 7-12. Each set of observations is matched separately. 


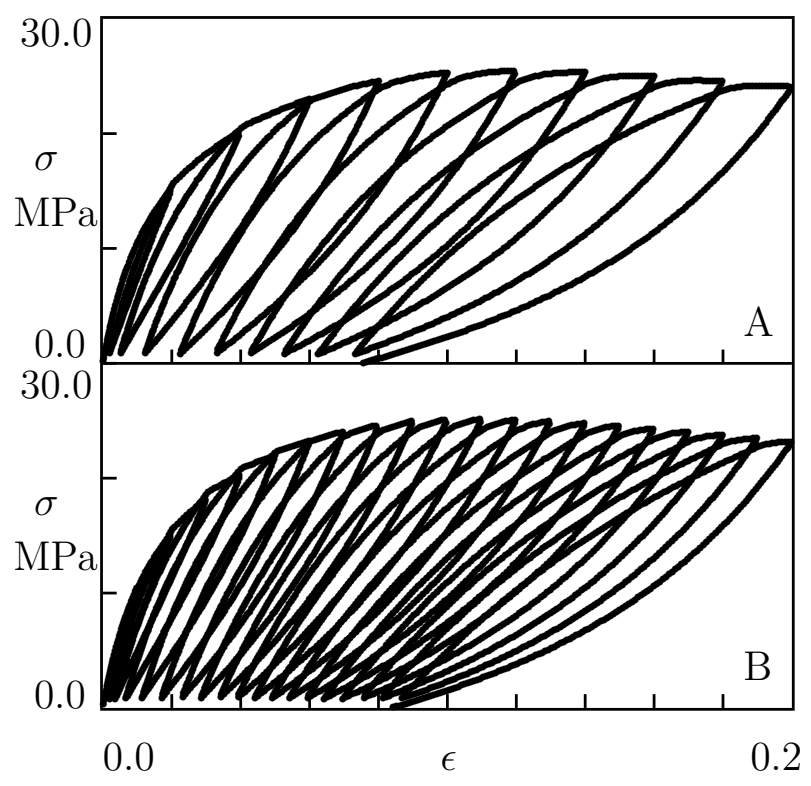

Figure 15: Stress $\sigma$ versus strain $\epsilon$. Solid lines: results of numerical simulation for cyclic tests with $\epsilon_{\max }(n)=0.2 n / N$. A: $N=10 ; \mathrm{B}: N=20$.

We begin with fitting the stress-strain diagram under stretching depicted in Figure 7 . We fix some intervals $\left[0, a^{\circ}\right],\left[0, R^{\circ}\right],\left[0, S^{\circ}\right]$, where $a_{1}, R_{1}$ and $S_{1}$ are located, and divide each of these intervals into $J=10$ sub-intervals by the points $a^{(i)}=i \Delta a$, $R^{(j)}=j \Delta R, S^{(l)}=l \Delta S$ with $\Delta a=a^{\circ} / J, \Delta R=R^{\circ} / J, \Delta S=S^{\circ} / J(i, j, l=0,1, \ldots, J-$ 1). For each triplet $\left\{a^{(i)}, R^{(j)}, S^{(l)}\right\}$, Eqs. (31), (33) are integrated numerically by the Runge-Kutta method with step $\Delta k=10^{-4}$. The modulus $\mu$ is calculated by the leastsquares technique from the condition of minimum of the function

$$
F=\sum_{m}\left[\sigma^{\exp }\left(k_{m}\right)-\sigma^{\text {num }}\left(k_{m}\right)\right]^{2}
$$

where summation is performed over all elongation ratios $k_{m}$ at which observations are reported, $\sigma^{\exp }$ is the engineering stress measured in the test, and $\sigma^{\text {num }}$ is given by Eq. (30). Coefficients $a_{1}, R_{1}$ and $S_{1}$ are found from the condition of minimum of $F$ on the set $\left\{a^{(i)}, R^{(j)}, S^{(l)}\right\}$. Afterwards, the initial intervals are replaced with new intervals $\left[a_{1}-\Delta a, a_{1}+\Delta a\right],\left[R_{1}-\Delta R, R_{1}+\Delta R\right],\left[S_{1}-\Delta S, S_{1}+\Delta S\right]$, and the calculations are repeated. The best-fit values of $\mu, a_{1}$, and $S_{1}$ read $\mu=0.57 \mathrm{GPa}, a_{1}=8.0, S_{1}=84.0$.

The experimental stress-strain curves depicted in Figures $7-12$ are matched by means of the above algorithm. Each path (stretching, unloading, and reloading) is fitted separately with the help of Eqs. (30), (31), (33). The best-fit adjustable parameters $R_{1}, R_{2}, R_{3}$ and $S_{2}, S_{3}$ are plotted versus specific plastic work $W_{\mathrm{p}}$ in Figures 13 and 14 ( $W_{\mathrm{p}}$ is determined by integration of Eq. (34) together with the stress-strain relations). The data are approximated by Eqs. (35) and (36) with coefficients calculated by the method of nonlinear regression.

Results of numerical simulation are presented in Figures 7-12 together with the experimental stress-strain diagrams. These figures demonstrate that the constitutive model correctly describes the viscoplastic behavior of polypropylene in cyclic tests with 


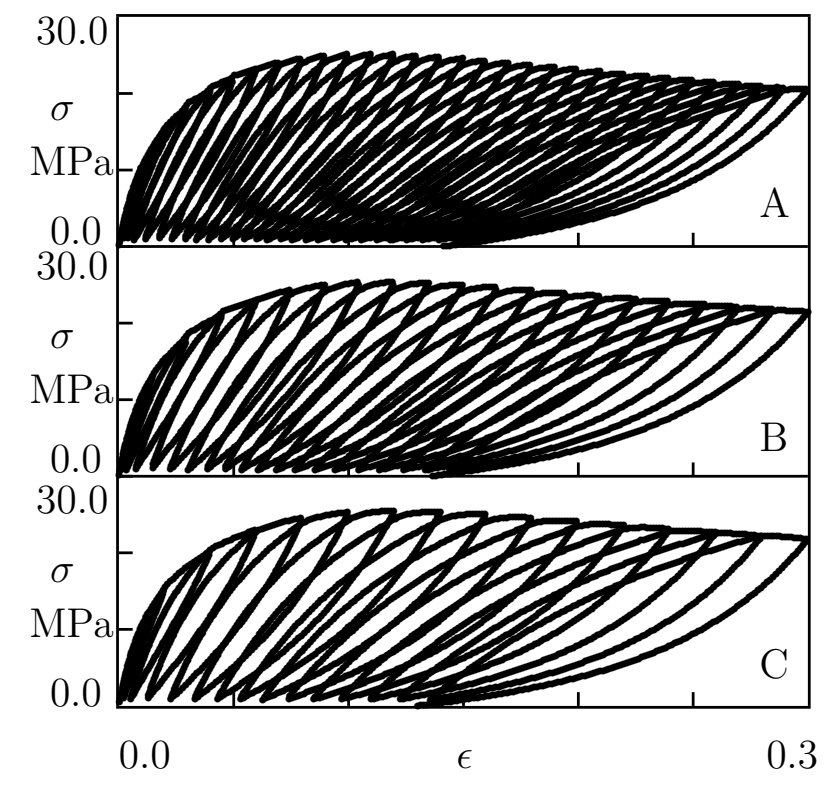

Figure 16: Stress $\sigma$ versus strain $\epsilon$. Solid lines: results of numerical simulation for cyclic tests with $\epsilon_{\max }(n)=0.3 n / N$. A: $N=30 ; \mathrm{B}: N=20 ; \mathrm{C}: N=15$.

increasing maximum elongation ratios.

\section{NUMERICAL SIMULATION}

To demonstrate ability of the model to predict the fading memory effect, integration of constitutive equations (30), (31), (33) is performed for uniaxial cyclic tests with increasing maximum strains and minimum stress $\sigma_{\min }=1 \mathrm{MPa}$. Simulation is conducted for tests with the following deformation programs (i) $\epsilon_{\max }(n)=0.2 n / N$ with $N=10$ and 20, and (ii) $\epsilon_{\max }(n)=0.3 n / N$ with $N=15,20$, and 30. Results of numerical analysis are depicted in Figures 15 and 16. These figures show that the model (i) predicts reasonably (without overshoots and undershoots) the stress-strain diagrams, (ii) describes adequately fading memory of deformation history (the last unloading paths of the stress-strain curves practically coincide).

\section{CONCLUSIONS}

Experimental stress-strain diagrams are reported for isotactic polypropylene in multistep cyclic tensile tests with monotonically increasing maximum strains. Observations demonstrate fading memory of deformation history: when specimens are subjected to two deformation programs that differ along the first $n-1$ cycles and coincide afterwards, the corresponding stress-strain curves become identical starting from the $n$th cycle.

A constitutive model is developed in cyclic viscoplasticity of semicrystalline polymers with finite deformations. Derivation of the constitutive equations is based on the following assumptions: (i) plastic deformation of a semicrystalline polymer is split 
into two components reflecting inelastic deformations in the crystalline and amorphous phases, (ii) strain energy density of a polymer equals the sum of mechanical energies stored in chains and the energy of their interaction, (iii) the rate-of-strain tensor for plastic flow in crystallites is proportional to that for macro-deformation, (iv) a flow rule for plastic deformation of the amorphous matrix is deduced from the Clausius-Duhem inequality.

The stress-strain relations involve eight adjustable parameters that are found by fitting the experimental data. It is demonstrated that the specific plastic work accounts adequately for the effect of damage accumulation on the mechanical response of semicrystalline polymers.

Ability of the model to describe the viscoplastic behavior of semicrystalline polymers in multi-step cyclic tests with various deformation programs and to predict the fading memory phenomenon is confirmed by numerical simulation.

\section{Acknowledgements}

The authors are thankful to Dr. N. Dusunceli for his help with performing mechanical tests.

\section{REFERENCES}

[1] Cantournet, S., Desmorat, R., Besson, J., "Mullins effect and cyclic stress softening of filled elastomers by internal sliding and friction thermodynamics model". Int. J. Solids Struct. 46, 2255-2264, 2009.

[2] Chagnon, G., Verron, E., Marckmann, G., Gornet, L., "Development of new constitutive equations for the Mullins effect in rubber using the network alteration theory". Int. J. Solids Struct. 43, 6817-6831, 2006.

[3] Diani, J., Fayolle, B., Gilormini, P., "A review on the Mullins effect". Eur. Polym. J. 45, 601-612, 2009.

[4] Drozdov, A. D., "Mullins' effect in semicrystalline polymers". Int. J. Solids Struct. 46, 3336-3345, 2009.

[5] Drozdov, A. D., "Cyclic strengthening of polypropylene under strain-controlled loading". Mater. Sci. Eng. A 528, 8781-8789, 2011.

[6] Drozdov, A. D., Christiansen, J. deC., "Mullins' effect in semicrystalline polymers: experiments and modeling". Meccanica 46, 359-370, 2011.

[7] Goktepe, S., Miehe, C., "A micro-macro approach to rubber-like materials. Part III: The micro-sphere model of anisotropic Mullins-type damage". J. Mech. Phys. Solids 53, 2259-2283, 2005.

[8] Itskov, M., Ehret, A. E., Kazakeviciute-Makovska, R., Weinhold, G. W., "A thermodynamically consistent phenomenological model of the anisotropic Mullins effect". ZAMM 90, 370-386, 2010.

[9] Qi, H. J., Boyce, M. C., "Constitutive model for stretch-induced softening of the stress-stretch behavior of elastomeric materials". J. Mech. Phys. Solids 52, 21872205,2004 . 